\title{
Fluence Rate in UV Photoreactor for Disinfection of Water: Isotropically Radiating Cylinder
}

\author{
Roman Ilinsky and Andrey Ulyanov \\ Joint Stock Company “Svarog”, Stromynka Street 18, Moscow 107014, Russia \\ Correspondence should be addressed to Roman Ilinsky; ilinsky_r@mail.ru
}

Received 28 February 2014; Accepted 29 August 2014; Published 10 November 2014

Academic Editor: Alírio Rodrigues

Copyright ( 2014 R. Ilinsky and A. Ulyanov. This is an open access article distributed under the Creative Commons Attribution License, which permits unrestricted use, distribution, and reproduction in any medium, provided the original work is properly cited.

\begin{abstract}
The calculation of fluence rate in the photochemical reactor using ultraviolet (UV) radiation for disinfection of water for the case, when a cylinder of infinite length is used as a light source, has been considered. Such a cylinder is filled with an isotropically radiating medium. The dependence of the fluent rate on the diameter of the radiating cylinder has been analytically analyzed. The limiting case when the diameter of the radiating cylinder tends to zero has been considered and the notion of "effective interval" has been introduced. Based on this notion, the comparison of fluence rates for the cylinders of finite and infinite lengths has been performed. In the calculations of fluence rate, it is advisable to use the Chebyshev method for the operations of numerical integration.
\end{abstract}

\section{Introduction}

In a normal design process, the last stage is the certification of the reactor for a certain set of operational parameters. In most situations, biodosimetric tests are used for this purpose $[1,2]$. If such certification fails, a certain part of the design process has to be repeated. The certification procedure and the necessary construction of a prototype unit are costly and timeconsuming. The available increasingly powerful numerical simulation techniques enable the designer to predict reactor performance under certain operating conditions without incurring the high cost of prototype construction and certification.

Numerical models simulating the disinfection process in a UV reactor involve fluence rate models to determine the distribution of intensity of UV radiation, the hydrodynamic models to predict motion paths of flow particles, and kinetic models of microbial inactivation [2-5]. The model of ultraviolet source (usually a lamp) is the significant element in the simulation of intensity distribution of UV radiation in a photoreactor $[4,5]$. Over the last few decades, several models for calculations of the distribution of lamp radiation intensity have been suggested [6-13]. In the simplest models, a lamp was represented by point sources which were arranged in a straight line. With the endless increase of a number of sources, one gets the model of a radiation source in a form of a line. In more complicated computer models, the radiation source is regarded as a three-dimensional body (usually such a body is a cylinder).

The goal of this work was to study analytically the model of radiation source in a form of infinite cylinder with regard to the distribution of fluence rate in a photoreactor. The radiation source in a form of a line is considered as the limiting case of the radiation source in a form of cylinder of infinitely small radius. In this study, we consider the case when this cylinder is filled with an isotropically radiating medium. A more simple case when the surface of the cylinder represents the diffuse radiator will be analyzed in the posterior paper.

\section{Nomenclature}

$$
\begin{aligned}
& E_{i}: \text { Fluence rate }\left[\mathrm{W} \cdot \mathrm{m}^{-2}\right] \\
& \ell: z \text { optical direction cosine of the ray (dimensionless) } \\
& n_{W}: \text { Refractive index of water (dimensionless) } \\
& n_{\text {Air }}: \text { Refractive index of air (dimensionless) } \\
& p: x \text { optical direction cosine of the ray (dimensionless) } \\
& q: y \text { optical direction cosine of the ray (dimensionless) } \\
& r_{\text {Air }}: \text { Radius of radiating cylinder [m] }
\end{aligned}
$$




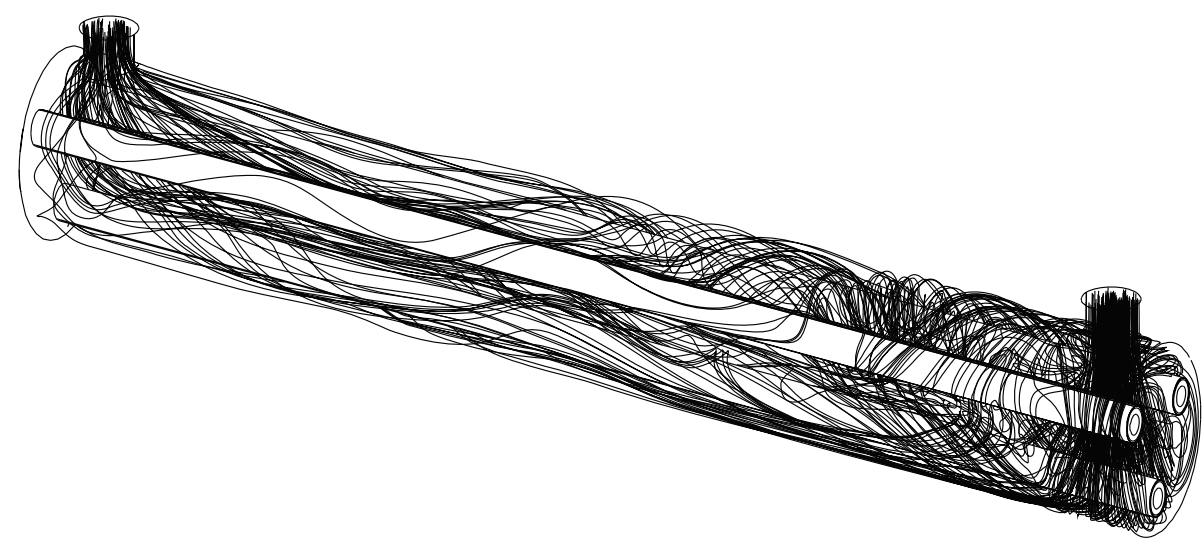

FIGURE 1: Experimental reactor of the "Svarog" Company. The figure shows motion paths of particles.

$$
\begin{aligned}
& r_{W}: \text { Radius of the water/air border }[\mathrm{m}] \\
& s: \text { Length }[\mathrm{m}] \\
& V: \text { Volume }\left[\mathrm{m}^{3}\right] \\
& x: \text { Rectangular coordinate }[\mathrm{m}] \\
& y: \text { Rectangular coordinate }[\mathrm{m}] \\
& z: \text { Rectangular coordinate }[\mathrm{m}] .
\end{aligned}
$$

\section{Greek Letters}

$\alpha$ : Absorption coefficient of water $\left[\mathrm{m}^{-1}\right]$

$\widehat{\beta}$ : Coefficient, which characterizes the volumetric density of the radiated flux $\left[\mathrm{W} \cdot \mathrm{m}^{-3}\right]$

$\beta$ : Radiated flux of radiating cylinder unit length $[\mathrm{W}$. $\mathrm{m}^{-1}$ ]

$\Theta$ : Angle between the portion of the ray path in water and axis $z$ [rad]

$\rho_{A}$ : Radius of the sphere with the center in point $A[\mathrm{~m}]$

$\sigma_{i}$ : Mean value of the area of cross section of the light tube $\left[\mathrm{m}^{2}\right]$

$\tau$ : Transmittance coefficient (dimensionless)

$\Phi:$ Radiation flux $[W]$

$\varphi$ : Angle between the projection of the portion of ray path in water on plane $x y$ and axis $y$ [rad]

$\Omega$ : Solid angle [sr].

\section{Basic Definitions}

A typical element of photoreactors, which are employed for water disinfection, consists of a quartz tube wherein a cylindrical lamp is placed. From the outside, this quartz tube is surrounded with water. The axes of the lamp and the quartz tube coincide (Figure 1). For the sake of simplifying the calculations of fluence rate, we will exclude from consideration

(i) the quartz casing between air and water,

(ii) the casing of the lamp, (iii) the reflection of radiation from the border between any two media with different refraction indexes,

(iv) the radiation from neighbouring lamps (for a multilamp reactor),

(v) the effect of reactor walls and of similar elements of the photoreactor.

The lamp is regarded as an infinitely long cylinder. This cylinder is evenly filled with a uniform substance radiating the energy. The refractive index of a given substance is equal to that of air. The region of space, which is filled with this substance, evenly radiates the flux in all directions:

$$
\Phi=4 \pi \widehat{\beta} V,
$$

where $\widehat{\beta}$ is the coefficient, which characterizes the volumetric density of the radiated flux, and $V$ is the volume of this region of space.

The fluence rate is calculated for point $A$ which is located in water (the medium under irradiation). Let us introduce the Descartes coordinate system $x y z$ (Figure 2 ). Axis $z$ coincides with the axis of radiating cylinder. Point $A$ lies on axis $y$. The direction of axis $y$ is selected so that $y$ coordinate of point $A$ should be negative $\left(y_{A}<0\right)$.

In the selected coordinate system, the water/air border is represented by the corresponding cylindrical surface:

$$
y^{2}+z^{2}=r_{W}^{2}
$$

where $r_{W}$ is the radius of the water/air border. The border of the radiating cylinder is described by equation

$$
y^{2}+z^{2}=r_{\text {Air }}^{2}
$$

where $r_{\text {Air }}$ is the radius of radiating cylinder. As follows from formula (1), the radiation flux from a portion of the radiating cylinder located between planes $z=z_{1}$ and $z=z_{2}$ is equal to

$$
\Phi_{\mathrm{cyl}}=\beta\left|z_{2}-z_{1}\right|
$$

where

$$
\beta=4 \pi^{2} r_{\text {Air }}^{2} \widehat{\beta}
$$




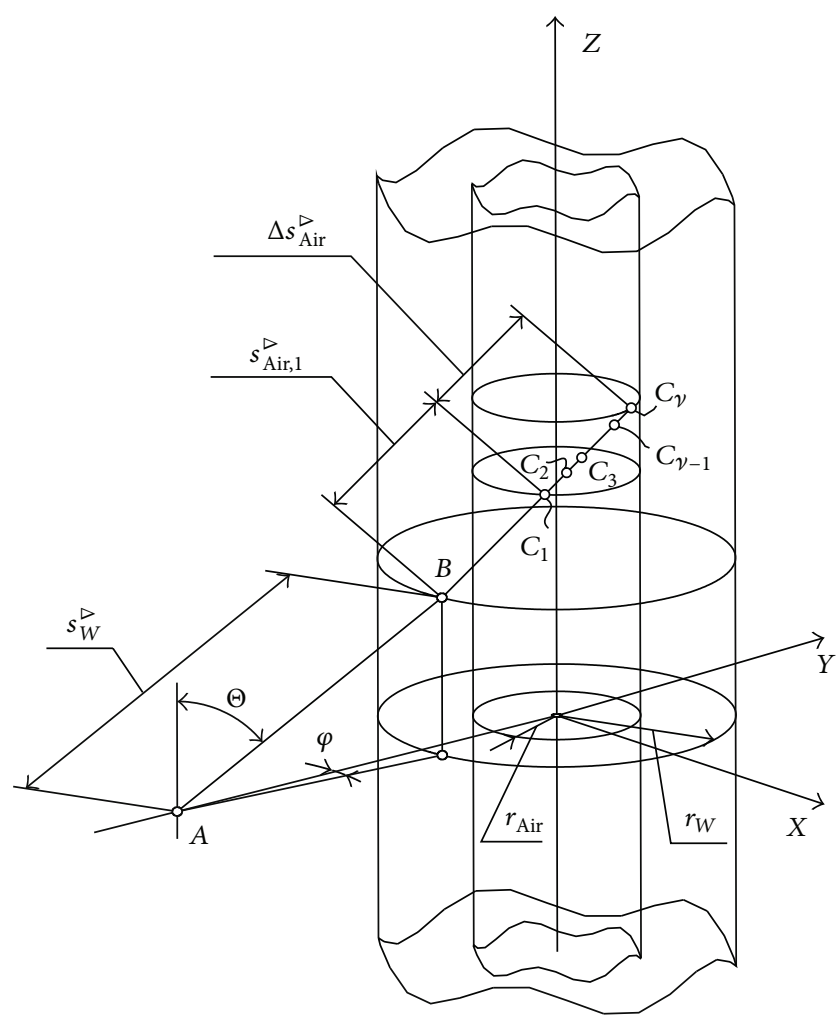

Figure 2: Explanatory draft of the calculations of fluence rate in point $A$.

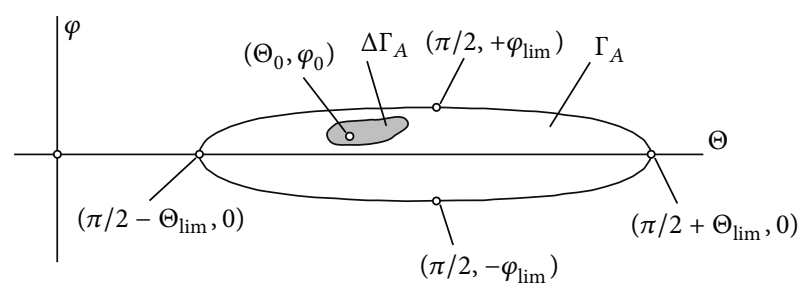

Figure 3: Region $\Gamma_{A}$ in the $\Theta-\varphi$ coordinate system.

\section{Calculations of Fluence Rate}

Every ray $\Re_{A}$ starting from point $A$ may be set by two angles, namely, by angle $\Theta$ between the portion of the ray path in water and axis $z$ and by angle $\varphi$ between the projection of the portion of ray path in water on plane $x y$ and axis $y$. The calculation of the ray path $\mathfrak{R}_{A}(\Theta, \varphi)$ is given in Appendix $A$. The conditions, under which the ray path $\Re_{A}(\Theta, \varphi)$ intersects the radiating cylinder, are stated in Appendix B. If at given angles $\Theta^{\prime}, \varphi^{\prime}$ the ray path $\Re_{A}\left(\Theta^{\prime}, \varphi^{\prime}\right)$ intersects the radiating cylinder, then in the system of $\Theta-\varphi$ coordinates point $\left(\Theta^{\prime}, \varphi^{\prime}\right)$ belongs to region $\Gamma_{A}$. Figure 3 shows the exemplary view of region $\Gamma_{A}$ in the $\Theta-\varphi$ coordinate system.

Note that $\Gamma_{A}$ is the closed and continuous region. Let us select a small uniform subregion $\Delta \Gamma_{A}$ inside $\Gamma_{A}$. The point with coordinates $\left(\Theta_{0}, \varphi_{0}\right)$ is the center of subregion $\Delta \Gamma_{A}$. Angles $\Theta_{0}$ and $\varphi_{0}$ define ray $\mathfrak{R}_{A}\left(\Theta_{0}, \varphi_{0}\right)$.

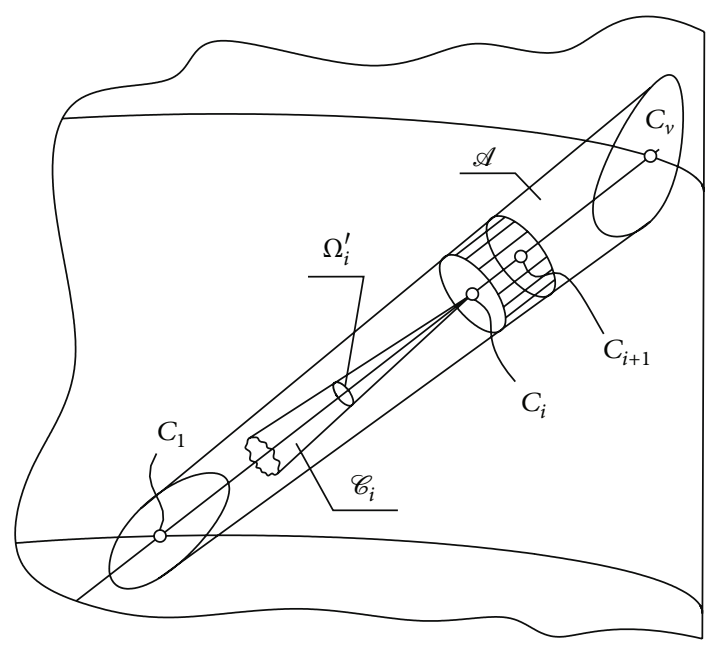

Figure 4: The portions of light tubes $\mathscr{A}$ and $\mathscr{C}_{i}$ in the radiating cylinder.

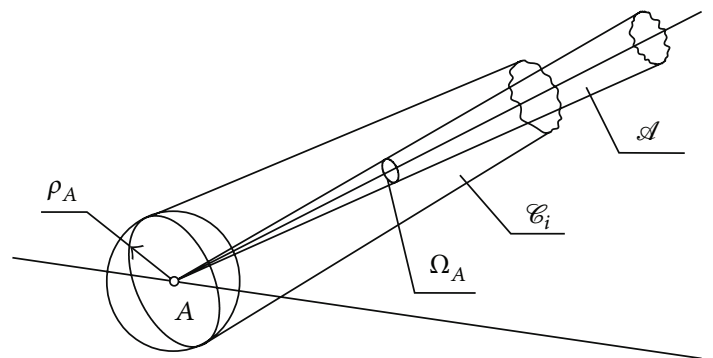

Figure 5: The portions of light tubes $\mathscr{A}$ and $\mathscr{C}_{i}$ near sphere $\left(A, \rho_{A}\right)$.

The path of ray $\mathfrak{R}_{A}\left(\Theta_{0}, \varphi_{0}\right)$ starts from point $A$ and intersects the water/air boundary (the surface (2)) in point $B$. Further, the path of ray $\mathfrak{R}_{A}\left(\Theta_{0}, \varphi_{0}\right)$ intersects the radiating cylinder (Figure 2). Let us divide the portion of ray path $\mathfrak{R}_{A}\left(\Theta_{0}, \varphi_{0}\right)$ in the cylinder into $v-1$ pieces of small lengths $C_{1} C_{2}, C_{2} C_{3}, \ldots, C_{\nu-1} C_{v}$ where $C_{1}$ and $C_{v}$ are the points of intersection of the reference ray with the radiating cylinder, $\left|C_{1} C_{\nu}\right|=\left|C_{1} C_{2}\right|+\left|C_{2} C_{3}\right|+\cdots+\left|C_{\nu-1} C_{\nu}\right|$.

Now, let us regard the light tube $\mathscr{A}$. This light tube represents the region of space in the system of $x y z$ coordinates. It involves all the points, through which the paths of rays $\mathfrak{R}_{A}\left(\Theta_{0}+\Delta \Theta, \varphi_{0}+\Delta \varphi\right)$ pass, when the condition $\left(\Theta_{0}+\Delta \Theta, \varphi_{0}+\right.$ $\Delta \varphi) \in \Delta \Gamma_{A}$ is fulfilled. As far as all rays $\Re_{A}\left(\Theta_{0}+\Delta \Theta, \varphi_{0}+\Delta \varphi\right)$ start from one point $A$, the initial portion of light tube $\mathscr{A}$ is a cone (Figure 4 ). The corresponding conical surface is the border of solid angle $\Omega_{A}$ with a vertex in point $A$. The value of this solid angle is

$$
\Omega_{A}=\iint_{\Delta \Gamma_{A}} \sin \Theta \mathrm{d} \Theta \mathrm{d} \varphi
$$

Taking into account that region $\Delta \Gamma_{A}$ has small sizes, the value of solid angle $\Omega_{A}$ may be assumed to be infinitesimal.

Let us draw planes through points $C_{1}, C_{2}, C_{3}, \ldots, C_{v-1}, C_{v}$ (Figure 5). These planes are perpendicular to straight line $C_{1} C_{v}$. Let $\sigma_{i}$ be the mean value of the area of cross section of the light tube $\mathscr{A}$ between planes $C_{i}$ and $C_{i+1}$. As $\Omega_{A} \rightarrow 0$, 
then $\sigma_{i} \rightarrow 0$. At $\left|C_{i} C_{i+1}\right| \rightarrow 0$ and $\sigma_{i} \rightarrow 0$, the volume of the portion of light tube $\mathscr{A}$ between planes $C_{i}$ and $C_{i+1}$ is equal to

$$
\Delta V_{i}=\sigma_{i}\left|C_{i} C_{i+1}\right| .
$$

As follows from relations (7), (1), and (5), the element of volume $\Delta V_{i}$ radiates evenly in all directions the flux

$$
\Delta \Phi_{i}=4 \pi \hat{\beta} \Delta V_{i}=\frac{\beta \Delta V_{i}}{\pi r_{\text {Air }}^{2}} .
$$

Let us circumscribe sphere $\left(A, \rho_{A}\right)$ with the center in point $A$ and infinitely small radius $\rho_{A}$. The radiation flux propagates from the portion of light tube $\mathscr{A}$ between planes $C_{i}$ and $C_{i+1}$ toward sphere $\left(A, \rho_{A}\right)$ through a light tube $\mathscr{C}_{i}$. The light tube $\mathscr{C}_{i}$ represents the region of space. It includes all the points through which the rays from the portion of light tube $\mathscr{A}$ between planes $C_{i}$ and $C_{i+1}$ pass to sphere $\left(A, \rho_{A}\right)$.

At $\left|C_{i} C_{i+1}\right| \rightarrow 0$ and $\sigma_{i} \rightarrow 0$, the radiating region, which is limited by planes $C_{i}$ and $C_{i+1}$ and light tube $\mathscr{A}$, may be considered as the point source. It is located in point $C_{i}$. The radiation flux from this point source is equal to $\Delta \Phi_{i}$ and is evenly distributed in all directions. In this case, the portion of light tube $\mathscr{C}_{i}$ in air is the cone with vertex in point $C_{i}$. The given conical surface is the border (the limiting element) of solid angle $\Omega_{i}^{\prime}$ with the vertex in point $C_{i}$. As the radiation flux $\Delta \Phi_{i}$ is evenly distributed over all directions (i.e., within solid angle $4 \pi$ ), the value of radiation flux within solid angle $\Omega_{i}^{\prime}$ is equal to

$$
\Delta \Phi_{i}^{\prime}=\frac{\Omega_{i}^{\prime}}{4 \pi} \Delta \Phi_{i}
$$

As follows from relations (8) and (9),

$$
\Delta \Phi_{i}^{\prime}=\frac{\beta}{4 \pi^{2} r_{\text {Air }}^{2}} \Omega_{i}^{\prime} \sigma_{i}\left|C_{i} C_{i+1}\right|
$$

In order to determine solid angle $\Omega_{i}^{\prime}$, we shall use the theorem Kirchhoff-Clausius-Straubel [14-17]. Based on this theorem, the relationship for light tubes $\mathscr{A}$ and $\mathscr{C}$ is as follows:

$$
\pi \rho_{A}^{2} n_{W}^{2} \Omega_{A}=\sigma_{i} \Omega_{i}^{\prime} .
$$

As a result of the substitution of relationship (11) into expression (8), we obtain

$$
\Delta \Phi_{i}^{\prime}=\frac{\beta\left|C_{i} C_{i+1}\right| \rho_{A}^{2} n_{W}^{2} \Omega_{A}}{4 \pi r_{\text {Air }}^{2}} .
$$

Taking into consideration the absorption of radiation in water, the flux of radiation falling onto sphere $\left(A, \rho_{A}\right)$ is expressed as

$$
\Phi_{i}^{\prime \prime}=\tau_{A B} \Phi_{i}^{\prime},
$$

where $\tau_{A B}$ is the transmittance coefficient for the portion of light tube $\mathscr{C}_{i}$ which is located in water. According to the Bouguer law [18],

$$
\tau_{A B}=\exp (-\alpha|A B|),
$$

where $\alpha$ is the absorption coefficient of water. (As a matter of convention, the transmittance of water, which fills a reactor, is characterized by transmittance $\tau^{*}$ of a water layer of known thickness $s^{*}$ (usually, $s^{*}=10 \mathrm{~mm}$ ); then, $\alpha=-\ln \tau^{*}\left(s^{*}\right) / s^{*}$.)

The element of volume $\Delta V_{i}$ creates the fluence rate $E_{i}$ in point $A$. Taking into account that, in the calculation of fluence rate $E_{i}$, radius $\rho_{A}$ should be taken as infinitesimal, the following expression should be valid:

$$
E_{i}=\lim _{\rho_{A} \rightarrow 0} \frac{\Phi_{i}^{\prime \prime}}{\pi \rho_{A}^{2}},
$$

where $\pi \rho_{A}^{2}$ is the area of the principle cross section of sphere $\left(A, \rho_{A}\right)$. As follows from relationship (15),

$$
E_{i}=\frac{\beta \exp (-\alpha|B A|)\left|C_{i} C_{i+1}\right| n_{W}^{2} \Omega_{A}}{4 \pi^{2} r_{\text {Air }}^{2}} .
$$

The fluence rate in point $A$ from the region of space, whose points simultaneously belong to the radiating cylinder and light tube $\mathscr{A}$, comprises the value

$$
\begin{aligned}
E_{\Delta \Gamma_{A}} & =\sum_{i=1}^{\nu-1} E_{i}=\frac{\beta \exp (-\alpha|B A|) n_{W}^{2} \Omega_{A}}{4 \pi^{2} r_{\text {Air }}^{2}} \sum_{i=1}^{\nu-1}\left|C_{i} C_{i+1}\right| \\
& =\frac{\beta \exp (-\alpha|B A|) n_{W}^{2} \Omega_{A}\left|C_{1} C_{\nu}\right|}{4 \pi^{2} r_{\text {Air }}^{2}} .
\end{aligned}
$$

As follows from relationships (6) and (17),

$$
E_{\Delta \Gamma_{A}}=\frac{\beta \exp (-\alpha|B A|) n_{W}^{2}\left|C_{1} C_{\nu}\right|}{4 \pi^{2} r_{\text {Air }}^{2}} \iint_{\Delta \Gamma_{A}} \sin \Theta \mathrm{d} \Theta \mathrm{d} \varphi .
$$

Based on relationship (A.4), we arrive at

$$
|B A|=\left|s_{W}^{\triangleright}\right|_{\Theta=\Theta_{0}, \varphi=\varphi_{0}}=\frac{-y_{A} \cos \varphi_{0}-\sqrt{r_{W}^{2}-y_{A}^{2} \sin ^{2} \varphi_{0}}}{\sin \Theta_{0}} .
$$

From relation (A.12), we get

$$
\begin{aligned}
\left|C_{1} C_{\nu}\right| & =\left|\Delta s_{\text {Air }}^{\triangleright}\right|_{\Theta=\Theta_{0}, \varphi=\varphi_{0}} \\
& =2 \frac{\sqrt{r_{\text {Air }}^{2}\left(1-n_{W}^{2} \cos ^{2} \Theta_{0}\right)-y_{A}^{2} n_{W}^{2} \sin ^{2} \varphi_{0} \sin ^{2} \Theta_{0}}}{1-n_{W}^{2} \cos ^{2} \Theta_{0}} .
\end{aligned}
$$

The substitution of expressions (19) and (20) into (18) gives

$$
\begin{aligned}
E_{\Delta \Gamma_{A}}= & \frac{n_{W}^{2} \beta}{2 \pi^{2} r_{\text {Air }}^{2}} \exp \left(-\alpha \frac{-y_{A} \cos \varphi_{0}-\sqrt{r_{W}^{2}-y_{A}^{2} \sin ^{2} \varphi_{0}}}{\sin \Theta_{0}}\right) \\
& \times \frac{\sqrt{r_{\text {Air }}^{2}\left(1-n_{W}^{2} \cos ^{2} \Theta_{0}\right)-y_{A}^{2} n_{W}^{2} \sin ^{2} \varphi_{0} \sin ^{2} \Theta_{0}}}{1-n_{W}^{2} \cos ^{2} \Theta_{0}} \\
& \times \iint_{\Delta \Gamma_{A}} \sin \Theta \mathrm{d} \Theta \mathrm{d} \varphi .
\end{aligned}
$$


As far as region $\Delta \Gamma_{A}$ has small sizes and the coordinates of its center are $\left(\Theta_{0}, \varphi_{0}\right)$, formula (21) may be transformed to the following view:

$$
\begin{aligned}
& E_{\Delta \Gamma_{A}} \\
& =\frac{n_{W}^{2} \beta}{2 \pi^{2} r_{\text {Air }}^{2}} \iint_{\Delta \Gamma_{A}} \exp \left(-\alpha \frac{-y_{A} \cos \varphi-\sqrt{r_{W}^{2}-y_{A}^{2} \sin ^{2} \varphi}}{\sin \Theta}\right) \\
& \quad \times \frac{\sqrt{r_{\text {Air }}^{2}\left(1-n_{W}^{2} \cos ^{2} \Theta\right)-y_{A}^{2} n_{W}^{2} \sin ^{2} \varphi \sin ^{2} \Theta}}{1-n_{W}^{2} \cos ^{2} \Theta}
\end{aligned}
$$$$
\times \sin \Theta \mathrm{d} \Theta \mathrm{d} \varphi
$$

The relationship for calculations of the complete fluence rate $E$ in point $A$ may be derived from (22) through the substitution of the integration field $\Delta \Gamma_{A}$ for $\Gamma_{A}$. Taking into account inequalities (B.5), which define the borders of region $\Gamma_{A}$, we obtain

$$
\begin{aligned}
E= & \frac{\beta n_{W}^{2}}{2 \pi^{2} r_{\text {Air }}^{2}} \\
& \times \int_{(\pi / 2)-\Theta_{\lim }}^{(\pi / 2)+\Theta_{\lim }} \int_{-\varphi_{\lim }}^{+\varphi_{\lim }} \exp \left(-\alpha \frac{-y_{A} \cos \varphi-\sqrt{r_{W}^{2}-y_{A}^{2} \sin ^{2} \varphi}}{\sin \Theta}\right) \\
& \times \frac{\sqrt{r_{\text {Air }}^{2}\left(1-n_{W}^{2} \cos ^{2} \Theta\right)-y_{A}^{2} n_{W}^{2} \sin ^{2} \varphi \sin ^{2} \Theta}}{1-n_{W}^{2} \cos ^{2} \Theta} \\
& \times \sin \Theta \mathrm{d} \varphi \mathrm{d} \Theta .
\end{aligned}
$$

Let

$$
\begin{aligned}
& \varphi=\arcsin \left(\frac{t}{\sqrt{1+t^{2}}}\right), \\
& \Theta=\pi-\arccos \left(\frac{u}{n_{W}}\right) .
\end{aligned}
$$

Then, (23) takes the following view:

$$
\begin{aligned}
E=\frac{n_{W} \beta}{2 \pi^{2} r_{\text {Air }}} & \\
& \times \int_{-1}^{+1} \int_{-t_{\lim }}^{+t_{\lim }} \sqrt{1-\frac{t^{2}}{t_{\lim }^{2}} \frac{1}{\left(1+t^{2}\right)^{3 / 2} \sqrt{1-u^{2}}}} \\
& \times \exp \left[-\alpha \frac{\left(-y_{A}-\sqrt{r_{W}^{2}+r_{W}^{2} t^{2}-y_{A}^{2} t^{2}}\right) n_{W}}{\sqrt{1+t^{2}} \sqrt{n_{W}^{2}-u^{2}}}\right]
\end{aligned}
$$

$\times \mathrm{d} t \mathrm{~d} u$,

where

$$
t_{\lim }=\frac{\sqrt{1-u^{2}} r_{\text {Air }}}{\sqrt{\left(n_{W}^{2}-u^{2}\right) y_{A}^{2}-\left(1-u^{2}\right) r_{\mathrm{Air}}^{2}}}
$$

Let us use the expansion in the Taylor series:

$$
\begin{aligned}
& \frac{\left(-y_{A}-\sqrt{r_{W}^{2}+r_{W}^{2} t^{2}-y_{A}^{2} t^{2}}\right) n_{W}}{\sqrt{1+t^{2}} \sqrt{n_{W}^{2}-u^{2}}} \\
& \approx \frac{\left(-y_{A}-r_{W}\right) n_{W}}{\sqrt{n_{W}^{2}-u^{2}}}+n_{W} \frac{r_{W} y_{A}+y_{A}^{2}}{2 r_{W} \sqrt{n_{W}^{2}-u^{2}}} t^{2} .
\end{aligned}
$$

After the substitution of expression (27) into (25), we obtain

$$
\begin{gathered}
E=\left(\frac{n_{W} \beta}{2 \pi^{2} r_{\text {Air }}}\right) \\
\times \int_{-1}^{+1} \int_{-\infty}^{+\infty} \exp \left[-\alpha \frac{\left(-y_{A}-r_{W}\right) n_{W}}{\sqrt{n_{W}^{2}-u^{2}}}\right] \\
\times \exp \left[-\alpha n_{W} \frac{r_{W} y_{A}+y_{A}^{2}}{2 r_{W} \sqrt{n_{W}^{2}-u^{2}}} t^{2}\right] h\left(\frac{t}{t_{\lim }}\right) \\
\times \frac{1}{\left(1+t^{2}\right)^{3 / 2} \sqrt{1-u^{2}}} \mathrm{~d} t \mathrm{~d} u
\end{gathered}
$$

where function

$$
h(\xi)= \begin{cases}0, & \text { at }|\xi|>1, \\ \sqrt{1-\xi^{2}}, & \text { at }|\xi| \leq 1\end{cases}
$$

describes the Wigner semicircle distribution.

\section{The Radiation Source in a Form of Line}

In the calculations of photoreactors, the cylindrical radiation source of finite sizes is very often substituted for the radiation source in a form of infinitely thin line. With that, the radiation fluxes from the portions of equal length of the cylindrical source and of the source in a form of infinitely thin line are regarded as equal.

The fluence rate in point $A$ from the source in a form of line represents the limiting value of expression (28) at $r_{\text {Air }} \rightarrow$ 0 :

$$
\begin{gathered}
E_{\text {line }}=\lim _{r_{\text {Air }} \rightarrow 0} \frac{\beta n_{W}}{2 \pi^{2} r_{\text {Air }}} \\
\times \int_{-1}^{+1} \int_{-\infty}^{+\infty} \exp \left[-\alpha \frac{\left(-y_{A}-r_{W}\right) n_{W}}{\sqrt{n_{W}^{2}-u^{2}}}\right] \\
\times \exp \left[-\alpha n_{W} \frac{r_{W} y_{A}+y_{A}^{2}}{2 r_{W} \sqrt{n_{W}^{2}-u^{2}}} t^{2}\right] h\left(\frac{t}{t_{\text {lim }}}\right) \\
\times \frac{1}{\left(1+t^{2}\right)^{3 / 2} \sqrt{1-u^{2}}} \mathrm{~d} t \mathrm{~d} u .
\end{gathered}
$$


Since $h(\xi)$ function is related to the Dirac delta function $\delta(\xi)$ by the relationship

$$
\delta(\xi)=\lim _{r_{\text {Air }} \rightarrow 0} \frac{2}{\pi r_{\text {Air }}} h\left(\frac{\xi}{r_{\text {Air }}}\right),
$$

then

$$
\begin{aligned}
E_{\text {line }}= & \frac{\beta n_{W}}{4 \pi\left|y_{A}\right|} \\
& \times \int_{-1}^{+1} \exp \left[-\alpha \frac{\left(-y_{A}-r_{W}\right) n_{W}}{\sqrt{n_{W}^{2}-u^{2}}}\right] \times \frac{1}{\sqrt{n_{W}^{2}-u^{2}}} \mathrm{~d} u .
\end{aligned}
$$

\section{Comparison of Values of Fluence Rate from the Radiation Source in a Form of Line and from the Cylindrical Radiation Source}

The integral in formula (25)

$$
\begin{aligned}
\mathfrak{I}=\int_{-t_{\lim }}^{+t_{\lim }} \exp & {\left[-\alpha n_{W} \frac{r_{W} y_{A}+y_{A}^{2}}{2 r_{W} \sqrt{n_{W}^{2}-u^{2}}} t^{2}\right] } \\
& \times h\left(\frac{t}{t_{\lim }}\right) \frac{1}{\left(1+t^{2}\right)^{3 / 2}} \mathrm{~d} t
\end{aligned}
$$

may be calculated only by numerical methods. One of such methods was suggested by Chebyshev [19]. By calculating integral (33) through the Chebyshev method, we get

$$
\begin{aligned}
& \mathfrak{s} \approx \mathfrak{S}\left\{\exp \left[-\alpha n_{W} \frac{r_{W} y_{A}+y_{A}^{2}}{2 r_{W} \sqrt{n_{W}^{2}-u^{2}}} t_{1}^{2}\right] \frac{1}{\left(1+t_{1}^{2}\right)^{3 / 2}}\right. \\
& +\exp \left[-\alpha n_{W} \frac{r_{W} y_{A}+y_{A}^{2}}{2 r_{W} \sqrt{n_{W}^{2}-u^{2}}} t_{2}^{2}\right] \frac{1}{\left(1+t_{2}^{2}\right)^{3 / 2}} \\
& +\exp \left[-\alpha n_{W} \frac{r_{W} y_{A}+y_{A}^{2}}{2 r_{W} \sqrt{n_{W}^{2}-u^{2}}} t_{3}^{2}\right] \\
& \left.\times \frac{1}{\left(1+t_{3}^{2}\right)^{3 / 2}}\right\}
\end{aligned}
$$

where

$$
\begin{gathered}
\mathfrak{S}=\frac{1}{3} \int_{-t_{\lim }}^{+t_{\lim }} h\left[\frac{t}{t_{\lim }}\right] \mathrm{d} t=\frac{\pi t_{\lim }}{6}, \\
t_{1}=-t_{3}=\sqrt{\frac{1}{2 \mathfrak{S}} \int_{-t_{\lim }}^{+t_{\lim }} h\left[\frac{t}{t_{\lim }}\right] t^{2} \mathrm{~d} t}=\sqrt{\frac{3}{8}} t_{\mathrm{lim}}, \\
t_{2}=0 .
\end{gathered}
$$

Let us expand expression (34) into series in powers of $r_{\text {Air }}$. If the terms beginning from $r_{\text {Air }}^{4}$ are rejected, then

$$
\begin{aligned}
\mathfrak{I} \approx & \frac{\pi}{2\left|y_{A}\right|} \cdot \sqrt{\frac{1-u^{2}}{n_{W}^{2}-u^{2}}} r_{\text {Air }} \\
& +0.2\left[r_{W} \sqrt{n_{W}^{2}-u^{2}}-\alpha n_{W} y_{A}^{2}-\alpha n_{W} y_{A} r_{W}\right] \\
& +\frac{\left(1-u^{2}\right)^{3 / 2}}{\left|y_{A}\right|^{3} r_{W}\left(n_{W}^{2}-u^{2}\right)} r_{\text {Air }}^{3} .
\end{aligned}
$$

The substitution of formula (36) into formula (25) allows us to get the expression which approximates the value of fluence rate in point $A$ :

$$
E \approx E_{\text {line }}+E_{r}
$$

where $E_{\text {line }}$ is the value of fluence rate in point $A$ from the source in a form of infinitely thin line (see formula (32))

$$
\begin{aligned}
E_{r}= & 0.2 \frac{\beta n_{W} r_{\text {Air }}^{2}}{2 \pi^{2}\left|y_{A}\right|^{3}} \\
& \times \int_{-1}^{+1}\left[1-\alpha n_{W} \frac{r_{W} y_{A}+y_{A}^{2}}{r_{W} \sqrt{n_{W}^{2}-u^{2}}}\right] \\
& \times \exp \left[-\alpha \frac{\left(-y_{A}-r_{W}\right) n_{W}}{\sqrt{n_{W}^{2}-u^{2}}}\right] \frac{1-u^{2}}{\sqrt{\left(n_{W}^{2}-u^{2}\right)^{3}}} \mathrm{~d} u .
\end{aligned}
$$

The ratio $E_{r} / E_{\text {line }}$ characterizes the relative accuracy of the fluence rate value under the substitution of the source in a form of infinitely thin line for the radiating cylinder. As follows from these formulas, the ratio takes the maximal value at $r_{\text {Air }} \rightarrow r_{W}$ and $y_{A} \rightarrow-r_{W}$. In this case,

$$
\frac{E_{r}}{E_{\text {line }}}=\frac{0.4}{\pi} \cdot \frac{\int_{-1}^{+1}\left(1-u^{2}\right)\left(n_{W}^{2}-u^{2}\right)^{-3 / 2} \mathrm{~d} u}{\int_{-1}^{+1}\left(n_{W}^{2}-u^{2}\right)^{-1 / 2} \mathrm{~d} u} .
$$

Based on formula (39), at $n_{W}=1.373$, which is the typical value for water, we have $E_{r} / E_{\text {line }} \approx 0.05$.

In the calculations of integrals in expressions (32) and (38), it is convenient to use the Chebyshev numerical method [19]. All formulas required for these operations are provided in Appendix C. It is easy to verify that, at $\alpha\left(y_{A}+r_{W}\right)=0$, the Chebyshev method gives the exact values of integrals in expressions (32) and (38).

\section{Effective Interval}

A lamp in a photoreactor cannot be of infinite length. Nevertheless, the formulas for the calculations of fluence rate at infinitely long cylindrical source of radiation are of practical importance. Let us regard the rays which start from point $A$ and form one and the same angle $\widetilde{\Theta}$ with axis $z$. The portions 


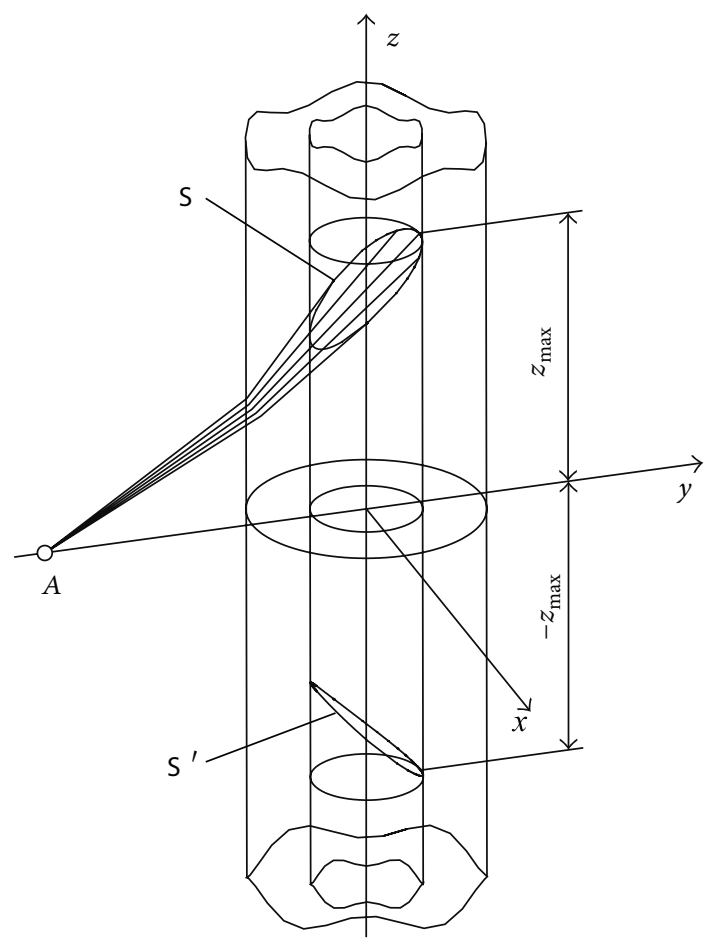

FIGURE 6: Explanatory draft for the definition of effective interval.

of paths of these rays in air lie on surface $\mathbb{\mathfrak { S }}$. The surface $\mathfrak{S}^{\prime}$ is symmetric with respect to surface $\mathbb{\subseteq}$ relative to plane $x y$ (Figure 6).

At $\pi / 2-\Theta_{\lim }<\widetilde{\Theta}<\pi / 2$, the maximal coordinate $z$ of the intersection of surface $\mathbb{S}$ with the radiating cylinder is equal to coordinate $z$ of the second (along the ray path) point of the intersection of ray $\Re_{A}(\widetilde{\Theta}, 0)$ with the cylindrical surface (3). As follows from formulas (A.5), (A.10), and (A.12), this coordinate is equal to

$$
z_{\max }=\frac{n_{W} \cos \widetilde{\Theta}\left(-y_{A}-r_{W}\right)}{\sqrt{n_{W}^{2}-n_{W}^{2} \cos ^{2} \widetilde{\Theta}}}+\frac{n_{W} \cos \widetilde{\Theta}\left(r_{\mathrm{Air}}+r_{W}\right)}{\sqrt{1-n_{W}^{2} \cos ^{2} \widetilde{\Theta}}} .
$$

Let the radiating volume $\mathfrak{V}$ be limited by the cylindrical surface (3), surface $\mathbb{S}$, and surface $\mathbb{S}^{\prime}$. To calculate the fluence rate in point $A$ from the radiating volume $\mathfrak{V}$, one may use formula (23) under the condition of substitution of integration limits

$$
\begin{aligned}
E^{*}= & \frac{\beta n_{W}^{2}}{2 \pi^{2} r_{\text {Air }}^{2}} \\
& \times \int_{+\widetilde{\Theta}}^{\pi-\widetilde{\Theta}} \int_{-\varphi_{\lim }}^{+\varphi_{\lim }} \exp \left(-\alpha \frac{-y_{A} \cos \varphi-\sqrt{r_{W}^{2}-y_{A}^{2} \sin ^{2} \varphi}}{\sin \Theta}\right) \\
& \times \frac{\sqrt{r_{\text {Air }}^{2}\left(1-n_{W}^{2} \cos ^{2} \Theta\right)-y_{A}^{2} n_{W}^{2} \sin ^{2} \varphi \sin ^{2} \Theta}}{1-n_{W}^{2} \cos ^{2} \Theta}
\end{aligned}
$$

$\times \sin \Theta \mathrm{d} \varphi \mathrm{d} \Theta$.
Similar to the procedure described in Section 6, it is possible to derive the approximate expression based on formula (41) for the calculation of fluence rate in point $A$ from the radiating volume $\mathfrak{V}$ :

$$
E^{*} \approx E_{\text {line }}^{*}+E_{r}^{*}
$$

where

$$
\begin{aligned}
E_{\text {line }}^{*}= & \frac{\beta n_{W}}{4 \pi\left|y_{A}\right|} \\
& \times \int_{-n_{W} \cos \widetilde{\Theta}}^{+n_{W} \cos \widetilde{\Theta}} \exp \left[-\alpha \frac{\left(-y_{A}-r_{W}\right) n_{W}}{\sqrt{n_{W}^{2}-u^{2}}}\right] \times \frac{1}{\sqrt{n_{W}^{2}-u^{2}}} \mathrm{~d} u, \\
E_{r}^{*}= & 0.2 \frac{\beta n_{W} r_{\text {Air }}^{2}}{2 \pi^{2}\left|y_{A}\right|^{3}} \\
& \times \int_{-n_{W} \cos \widetilde{\Theta}}^{+n_{W} \cos \widetilde{\Theta}}\left[1-\alpha n_{W} \frac{r_{W} y_{A}+y_{A}^{2}}{r_{W} \sqrt{n_{W}^{2}-u^{2}}}\right] \\
& \times \exp \left[-\alpha \frac{\left(-y_{A}-r_{W}\right) n_{W}}{\sqrt{n_{W}^{2}-u^{2}}}\right] \times \frac{1-u^{2}}{\sqrt{\left(n_{W}^{2}-u^{2}\right)^{3}}} \mathrm{~d} u .
\end{aligned}
$$

Let us find such value of $\widetilde{\Theta}$, at which the following condition is fulfilled:

$$
E-E^{*} \leq \mu E,
$$

where $\mu$ is a given value. Based on expressions (37) and (42), inequality (44) may be presented in the form:

$$
E_{\text {line }}+E_{r}-E_{\text {line }}^{*}-E_{r}^{*} \leq \mu\left(E_{\text {line }}+E_{r}\right) .
$$

At small values of $\mu$, condition (44) is fulfilled in the case when $n_{W} \cos \widetilde{\Theta} \approx 1$. With an accuracy of values of the second order of smallness relative to $\left(1-n_{W} \cos \widetilde{\Theta}\right)$, formula (43) acquires the view:

$$
\begin{gathered}
E_{\text {line }}^{*}=E_{\text {line }}-\exp \left[-\alpha \frac{\left(-y_{A}-r_{W}\right) n_{W}}{\sqrt{n_{W}^{2}-1}}\right] \\
\times \frac{\beta n_{W}^{2}}{2\left|y_{A}\right| \pi \sqrt{n_{W}^{2}-1}}\left(1-n_{W} \cos \widetilde{\Theta}\right), \\
E_{r}^{*}=E_{r} .
\end{gathered}
$$

It is seen from these formulas that inequality (45) is transformed into equality $E_{\text {line }}+E_{r}-E_{\text {line }}^{*}-E_{r}^{*}=\mu E_{\text {line }}+E_{r}$ at

$$
\begin{aligned}
\cos \widetilde{\Theta}= & \frac{1}{n_{W}} \\
& -\frac{2 \mu\left(E_{\text {line }}+E_{r}\right) \pi\left|y_{A}\right| \sqrt{n_{W}^{2}-1}}{n_{W}^{2} \beta \exp \left[-\alpha\left(\left(-y_{A}-r_{W}\right) n_{W} / \sqrt{n_{W}^{2}-1}\right)\right]} .
\end{aligned}
$$




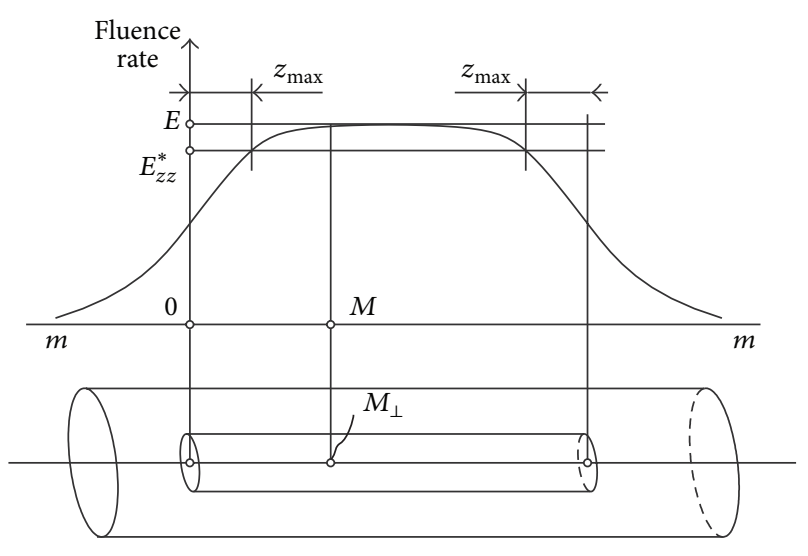

Figure 7: The fluence rate from the cylindrical source of finite length. The plot is given without scale compliance.

Upon the substitution of the obtained value of $\cos \widetilde{\Theta}$ into formula (40), we may find the value of distance $z_{\max }$.

The portion of radiating cylinder, which is limited by planes $z=z_{\max }$ and $z=-z_{\max }$, creates the fluence rate $E_{z z}^{*}=E^{*}+2 E_{\mathfrak{\subseteq}_{z}}^{*}$, in point $A$, where $E_{\mathfrak{\subseteq}_{z}}^{*}$ is the fluence rate from the portion of radiating cylinder, which is limited by plane $z=z_{\max }$ and surface $\mathfrak{S}$. As $E_{\mathfrak{S}_{z}}^{*}>0$, then $E_{z z}^{*}>E^{*}$. It is evident that $E_{z z}^{*}<E$. Hence, $E>E_{z z}^{*}>E^{*}$, or

$$
E>E_{z z}^{*}>(1-\mu) E
$$

As follows from inequality (48), the fluence rate from the portion of radiating cylinder, which is limited by planes $z=$ $z_{\max }$ and $z=-z_{\max }$, and the fluence rate from the infinite radiating cylinder differ by most $\mu E$. Based on this fact, we will name the interval $\left[-z_{\max } ; z_{\max }\right]$ as the effective interval.

Let us use the concept of the effective interval for the estimation of the fluence rate along the line $m-m$, which is parallel to the axis of the radiating cylinder of finite length (Figure 7), and calculate the values of $E_{\text {line }}, E_{r}$, and $z_{\max }$ by means of formulas (32), (38), and (40). Let point $M$ be located on the axis of radiating cylinder. Point $M_{\perp}$ is the projection of point $M$ onto the axis of the cylinder. If each of the distances from point $M_{\perp}$ to the face planes of the cylinder is larger than a half length of the effective interval (i.e., $z_{\max }$ ), then the fluence rate in point $M$ cannot be greater than $E$ and cannot be smaller than $(1-\mu) E$. If the length of radiating cylinder is greater than the effective interval, then the plot of fluence rate along the line $m-m$ has the form of trapezium with curvilinear lateral sides. With that, the length of the plot portion where the fluence rate is greater than $(1-\mu) E$ and smaller than $E$ is equal to the difference between the length of the radiating cylinder and the length of effective interval.

The data of calculations and experiments presented in studies [3-6] confirm that the above described plot of the fluence rate is characterized by the trapezium form with curvilinear lateral sides.

\section{Example}

The calculations of fluence rate are performed at $r_{W}=16 \mathrm{~mm}$. The refractive index of water under irradiation depends on a wavelength, temperature, and admixtures dissolved in water [20]. In all calculations, the refractive index of water under irradiation is assumed to be equal to $n_{W}=1.373$.

For every set of parameters $y_{A}, r_{\text {Air }}$, and $\alpha$, the values of fluence rate were calculated

(i) by means of the ray-trace procedure,

(ii) by means of formulas (37), (32), and (38).

In the calculations of fluence rate by the method of ray tracing, the rectangular net was set in the $\Theta-\varphi$ coordinate system. The centers of net meshes were located in points $\left(\widehat{\Theta}_{i}, \widehat{\varphi}_{j}\right)$, where $\widehat{\Theta}_{i}=\arccos [(1 / 1024)(j+1 / 2)], i=0, \pm 1, \pm 2, \pm 3, \ldots$, and $\widehat{\varphi}_{j}=(2 \pi / 1024)(j+1 / 2), j=0, \pm 1, \pm 2, \pm 3, \ldots$. The sizes of the net knowingly exceeded region $\Gamma_{A}$. Every net mesh had a corresponding ray $\Re_{A}\left(\widehat{\Theta}_{i}, \widehat{\varphi}_{j}\right)$. The procedure of calculations of ray path $\Re_{A}\left(\widehat{\Theta}_{i}, \widehat{\varphi}_{j}\right)$ is considered in Appendix A. If ray $\mathfrak{R}_{A}\left(\widehat{\Theta}_{i}, \widehat{\varphi}_{j}\right)$ intersects the radiating cylinder (see Appendix A), then the mesh with center in point $\left(\widehat{\Theta}_{i}, \widehat{\varphi}_{j}\right)$ is regarded as subregion $\Delta \Gamma_{A}$ of region $\Gamma_{A}$, and the fluence rate is calculated by formula (21). The fluence rate in point $A$ is equal to the sum of fluence rates from all meshes belonging to region $\Gamma_{A}$.

In the calculations of the fluence rates according to formulas (37), (32), and (38), the integrals were determined

(i) with the use of a Fortran subroutine for the calculation of define integral according to the Simpson generalized quadrature rule with the specified relative accuracy of 0.01 ;

(ii) with the use of the Chebyshev formulas (C.2) and (C.3).

The calculated values of fluence rate are summarized in Tables 1 and 2. There is a good agreement between all data of these calculations.

Tables 3 and 4 present the values of the effective interval lengths.

\section{Conclusions}

The calculations of fluence rate in the ultraviolet photoreactor for water disinfection have been considered for the case when a cylinder of infinite length is the radiation source. This cylinder is filled with an isotropically radiating medium. The dependence of fluence rate on the diameter of radiating cylinder has been analytically analyzed. The radiation source in a form of line is regarded as the limiting case when the diameter of radiating cylinder tends to zero. The comparison of values of the fluence rate from the radiation source in a form of line and from the cylindrical source shows that, at the value of refractive index of the medium under irradiation equal to 1.373 , the difference between fluence rates from these sources is smaller than $1 \%$. The notion of "effective interval" 
TABLE 1: The values of fluence rate. The transmittance of $10 \mathrm{~mm}$ thick water layer is $60 \%$.

\begin{tabular}{|c|c|c|c|c|c|c|c|}
\hline \multirow{2}{*}{$y_{A}[\mathrm{~mm}]$} & \multirow{2}{*}{$\begin{array}{l}r_{\mathrm{Air}} \\
{[\mathrm{mm}]}\end{array}$} & \multirow{2}{*}{$\begin{array}{l}\text { The ray-trace procedure } \\
\qquad E / \beta\left[\mathrm{m}^{-1}\right]\end{array}$} & \multicolumn{3}{|c|}{ The integration by the Simpson method } & \multicolumn{2}{|c|}{ The integration by the Chebyshev method } \\
\hline & & & $\begin{array}{c}\left(E_{\text {line }}+E_{r}\right) / \beta \\
{\left[\mathrm{m}^{-1}\right]}\end{array}$ & $\begin{array}{c}E_{\text {line }} / \beta \\
{\left[\mathrm{m}^{-1}\right]}\end{array}$ & $E_{r} / \beta\left[\mathrm{m}^{-1}\right]$ & $E_{\text {line }} / \beta\left[\mathrm{m}^{-1}\right]$ & $E_{r} / \beta\left[\mathrm{m}^{-1}\right]$ \\
\hline-16.0 & 5.0 & 11.199 & 11.197 & 11.143 & 0.054 & 11.143 & 0.054 \\
\hline-16.0 & 10.0 & 11.373 & 11.358 & 11.143 & 0.215 & 11.143 & 0.215 \\
\hline-16.0 & 15.0 & 11.712 & 11.627 & 11.143 & 0.484 & 11.143 & 0.484 \\
\hline-21.0 & 5.0 & 6.370 & 6.370 & 6.359 & 0.011 & 6.371 & 0.012 \\
\hline-21.0 & 10.0 & 6.406 & 6.404 & 6.359 & 0.046 & 6.371 & 0.046 \\
\hline-21.0 & 15.0 & 6.466 & 6.462 & 6.359 & 0.103 & 6.371 & 0.104 \\
\hline-26.0 & 5.0 & 3.854 & 3.851 & 3.851 & 0.001 & 3.863 & 0.001 \\
\hline-26.0 & 10.0 & 3.854 & 3.854 & 3.851 & 0.003 & 3.863 & 0.003 \\
\hline-26.0 & 15.0 & 3.854 & 3.857 & 3.851 & 0.007 & 3.863 & 0.007 \\
\hline-31.0 & 5.0 & 2.424 & 2.422 & 2.424 & -0.002 & 2.434 & -0.002 \\
\hline-31.0 & 10.0 & 2.416 & 2.416 & 2.424 & -0.008 & 2.434 & -0.008 \\
\hline-31.0 & 15.0 & 2.404 & 2.406 & 2.424 & -0.017 & 2.434 & -0.017 \\
\hline-36.0 & 5.0 & 1.565 & 1.566 & 1.568 & -0.002 & 1.575 & -0.002 \\
\hline-36.0 & 10.0 & 1.559 & 1.559 & 1.568 & -0.009 & 1.575 & -0.009 \\
\hline-36.0 & 15.0 & 1.546 & 1.547 & 1.568 & -0.021 & 1.575 & -0.021 \\
\hline-41.0 & 5.0 & 1.033 & 1.033 & 1.035 & -0.002 & 1.040 & -0.002 \\
\hline-41.0 & 10.0 & 1.027 & 1.027 & 1.035 & -0.008 & 1.040 & -0.008 \\
\hline-41.0 & 15.0 & 1.016 & 1.017 & 1.035 & -0.018 & 1.040 & -0.018 \\
\hline
\end{tabular}

TABLE 2: The values of fluence rate. The transmittance of $10 \mathrm{~mm}$ thick water layer is $80 \%$.

\begin{tabular}{|c|c|c|c|c|c|c|c|}
\hline \multirow{2}{*}{$\begin{array}{l}y_{A} \\
{[\mathrm{~mm}]}\end{array}$} & \multirow{2}{*}{$\begin{array}{l}r_{\text {Air }} \\
{[\mathrm{mm}]}\end{array}$} & \multirow{2}{*}{$\begin{array}{l}\text { The ray-trace procedure } \\
\qquad E / \beta\left[\mathrm{m}^{-1}\right]\end{array}$} & \multicolumn{3}{|c|}{ The integration by the Simpson method } & \multicolumn{2}{|c|}{ The integration by the Chebyshev method } \\
\hline & & & $\begin{array}{c}\left(E_{\text {line }}+E_{r}\right) / \beta \\
{\left[\mathrm{m}^{-1}\right]}\end{array}$ & $\begin{array}{c}E_{\text {line }} / \beta \\
{\left[\mathrm{m}^{-1}\right]}\end{array}$ & $E_{r} / \beta\left[\mathrm{m}^{-1}\right]$ & $E_{\text {line }} / \beta\left[\mathrm{m}^{-1}\right]$ & $E_{r} / \beta\left[\mathrm{m}^{-1}\right]$ \\
\hline-16.0 & 5.0 & 11.199 & 11.197 & 11.143 & 0.054 & 11.143 & 0.054 \\
\hline-16.0 & 10.0 & 11.373 & 11.358 & 11.143 & 0.215 & 11.143 & 0.215 \\
\hline-16.0 & 15.0 & 11.712 & 11.627 & 11.143 & 0.484 & 11.143 & 0.484 \\
\hline-21.0 & 5.0 & 7.499 & 7.499 & 7.482 & 0.018 & 7.489 & 0.018 \\
\hline-21.0 & 10.0 & 7.555 & 7.553 & 7.482 & 0.071 & 7.489 & 0.071 \\
\hline-21.0 & 15.0 & 7.653 & 7.641 & 7.482 & 0.160 & 7.489 & 0.160 \\
\hline-26.0 & 5.0 & 5.337 & 5.332 & 5.326 & 0.006 & 5.336 & 0.006 \\
\hline-26.0 & 10.0 & 5.352 & 5.350 & 5.326 & 0.024 & 5.336 & 0.024 \\
\hline-26.0 & 15.0 & 5.381 & 5.380 & 5.326 & 0.054 & 5.336 & 0.054 \\
\hline-31.0 & 5.0 & 3.944 & 3.940 & 3.938 & 0.002 & 3.948 & 0.002 \\
\hline-31.0 & 10.0 & 3.945 & 3.945 & 3.938 & 0.006 & 3.948 & 0.006 \\
\hline-31.0 & 15.0 & 3.951 & 3.952 & 3.938 & 0.014 & 3.948 & 0.014 \\
\hline-36.0 & 5.0 & 2.990 & 2.990 & 2.991 & 0.000 & 3.000 & 0.000 \\
\hline-36.0 & 10.0 & 2.990 & 2.990 & 2.991 & -0.001 & 3.000 & -0.001 \\
\hline-36.0 & 15.0 & 2.987 & 2.988 & 2.991 & -0.002 & 3.000 & -0.002 \\
\hline-41.0 & 5.0 & 2.314 & 2.315 & 2.316 & -0.001 & 2.324 & -0.001 \\
\hline-41.0 & 10.0 & 2.312 & 2.312 & 2.316 & -0.004 & 2.324 & -0.004 \\
\hline-41.0 & 15.0 & 2.307 & 2.307 & 2.316 & -0.009 & 2.324 & -0.009 \\
\hline
\end{tabular}


TABLE 3: The values of $z_{\max }$. The transmittance of $10 \mathrm{~mm}$ thick water layer is $60 \%$.

\begin{tabular}{lccc}
\hline$y_{A}[\mathrm{~mm}]$ & $r_{\text {Air }}[\mathrm{mm}]$ & $\begin{array}{c}2 z_{\max }(\mu=0.15) \\
{[\mathrm{mm}]}\end{array}$ & $\begin{array}{c}2 z_{\max }(\mu=0.05) \\
{[\mathrm{mm}]}\end{array}$ \\
\hline-16.00 & 5.00 & 79.55 & 146.82 \\
-16.00 & 10.00 & 97.64 & 180.40 \\
-16.00 & 15.00 & 114.79 & 212.44 \\
-21.00 & 5.00 & 84.02 & 150.40 \\
-21.00 & 10.00 & 101.74 & 183.39 \\
-21.00 & 15.00 & 119.09 & 215.79 \\
-26.00 & 5.00 & 88.16 & 153.89 \\
-26.00 & 10.00 & 105.27 & 185.87 \\
-26.00 & 15.00 & 122.33 & 217.79 \\
-31.00 & 5.00 & 91.97 & 157.33 \\
-31.00 & 10.00 & 108.37 & 188.15 \\
-31.00 & 15.00 & 124.93 & 219.23 \\
-36.00 & 5.00 & 95.42 & 160.73 \\
-36.00 & 10.00 & 111.07 & 190.33 \\
-36.00 & 15.00 & 127.02 & 220.38 \\
-41.00 & 5.00 & 98.47 & 164.07 \\
-41.00 & 10.00 & 113.36 & 192.43 \\
-41.00 & 15.00 & 128.65 & 221.37 \\
\hline
\end{tabular}

TABLE 4: The values of $z_{\max }$. The transmittance of $10 \mathrm{~mm}$ thick water layer is $80 \%$.

\begin{tabular}{lccc}
\hline$y_{A}[\mathrm{~mm}]$ & $r_{\text {Air }}[\mathrm{mm}]$ & $\begin{array}{c}2 z_{\max }(\mu=0.15) \\
{[\mathrm{mm}]}\end{array}$ & $\begin{array}{c}2 z_{\max }(\mu=0.05) \\
{[\mathrm{mm}]}\end{array}$ \\
\hline-16.00 & 5.00 & 79.55 & 146.82 \\
-16.00 & 10.00 & 97.64 & 180.40 \\
-16.00 & 15.00 & 114.79 & 212.44 \\
-21.00 & 5.00 & 86.29 & 153.98 \\
-21.00 & 10.00 & 104.42 & 187.64 \\
-21.00 & 15.00 & 122.07 & 220.52 \\
-26.00 & 5.00 & 92.85 & 161.02 \\
-26.00 & 10.00 & 110.81 & 194.39 \\
-26.00 & 15.00 & 128.53 & 227.39 \\
-31.00 & 5.00 & 99.26 & 167.98 \\
-31.00 & 10.00 & 116.95 & 200.92 \\
-31.00 & 15.00 & 134.56 & 233.73 \\
-36.00 & 5.00 & 105.51 & 174.89 \\
-36.00 & 10.00 & 122.90 & 207.33 \\
-36.00 & 15.00 & 140.30 & 239.79 \\
-41.00 & 5.00 & 111.61 & 181.76 \\
-41.00 & 10.00 & 128.67 & 213.65 \\
-41.00 & 15.00 & 145.81 & 245.67 \\
\hline
\end{tabular}

has been introduced. Based on this notion, the conditions, under which the fluence rate from the infinitely long cylinder and from the cylinder of finite length differs by indefinitely

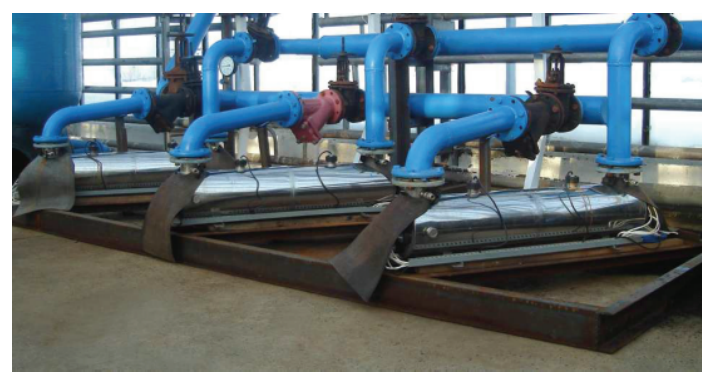

FIGURE 8: Multilamp reactors of "Lasur" series.

small preliminary specified value, have been formulated. It has been shown that in the calculations of fluence rate the use of the Chebyshev method for numerical integration is expedient.

This paper is the result of investigations which have been performed and sponsored by the JSC "Svarog" with the purpose of creating high-performance photochemical reactors of "Lasur" series designed for water disinfection (see Figure 8). The results of investigations reported in the present paper are actively employed in the JSC "Svarog." Thus, taking into account that the diameter of radiation source slightly affects the fluence rate, the ratio (radiation flux)/(length of lamp) has been accepted as the main criterion for the of photoreactor lamps. The estimation of a variation in the fluence rate for the direction parallel to the lamp made on basis of the "effective interval" notion is useful for the choice of proper places for devices of water input and output and arrangement of the sources of ultrasound in the photoreactor.

For more accurate computation of the operational characteristics of photoreactors, the JSC Svarog uses the complicated computer simulation. The models are based on the calculations of fluence rate in points of particle pathways (see Figure 1). These calculations take into account such factors as the impact of quartz casing between air and water, the reflection of radiation from a border between two media with different refractive indexes, the mutual effect of neighbouring lamps (for multilamp reactors), and the effects of walls and similar design elements of photoreactor. With that, several lamp models are used. For the final evaluation of operating performance of photoreactors, the JSC "Svarog" uses biodosimetric tests [21].

\section{Appendices}

\section{A. Calculation of Ray Path}

Let us assume that a ray starts from point $A$. The coordinates of point $A$ are $x=0, y=y_{A}$, and $z=0$. Then, the portion of ray path in water is described by equations

$$
x=\frac{p_{W} s_{W}}{n_{W}}, \quad y=y_{A}+\frac{q_{W} s_{W}}{n_{W}}, \quad z=\frac{\ell_{W} s_{W}}{n_{W}},
$$


where

$$
\begin{gathered}
p_{W}=n_{W} \sin \Theta \sin \varphi, \quad q_{W}=n_{W} \sin \Theta \cos \varphi, \\
\ell_{W}=n_{W} \cos \Theta
\end{gathered}
$$

are the optical direction cosines of the portion of ray path in water, $\Theta$ is the angle between the portion of ray path in water and axis $z, \varphi$ is the angle between the projection of the entry path portion on plane $x y$ and axis $y, n_{W}$ is the refractive index, and $s_{W}$ is the distance from point $A$ to the point with coordinates $x, y$, and $z$.

Assume that, at $s_{W}=s_{W}^{\triangleright}$, the ray intersects the cylindrical surface (2). Then,

$$
s_{W}^{\triangleright}=n_{W} \frac{-y_{A} q_{W}-\sqrt{r_{W}^{2}\left(p_{W}^{2}+q_{W}^{2}\right)-y_{A}^{2} p_{W}^{2}}}{p_{W}^{2}+q_{W}^{2}} .
$$

By the substitution of expressions (A.1) into formula (2), we get

$$
s_{W}^{\triangleright}=\frac{-y_{A} \cos \varphi-\sqrt{r_{W}^{2}-y_{A}^{2} \sin ^{2} \varphi}}{\sin \Theta} .
$$

The portion of ray path in air is described by equations

$$
\begin{aligned}
& x=x^{\prime}+\frac{p_{\mathrm{Air}} s_{\mathrm{Air}}}{n_{\mathrm{Air}}}, \\
& y=y^{\prime}+\frac{q_{\mathrm{Air}} s_{\mathrm{Air}}}{n_{\mathrm{Air}}} \\
& z=z^{\prime}+\frac{\ell_{\mathrm{Air}} s_{\mathrm{Air}}}{n_{\mathrm{Air}}}
\end{aligned}
$$

where $x^{\prime}=p_{W} s_{W}^{\triangleright} / n_{W}=\left(-y_{A} \cos \varphi-\sqrt{r_{W}^{2}-y_{A}^{2} \sin ^{2} \varphi}\right) \sin \varphi$, $y^{\prime}=y_{A}+q_{W} s_{W}^{\triangleright} / n_{W}=y_{A}+\left(-y_{A} \cos \varphi-\sqrt{r_{W}^{2}-y_{A}^{2} \sin ^{2} \varphi}\right)$ $\cos \varphi$, and $z^{\prime}=\ell_{W} s_{W}^{\triangleright} / n_{W}$ the coordinates of the point $B$ of intersection of the ray with cylindrical surface; $s_{\text {Air }}$ is the distance from the point with coordinates $x^{\prime}, y^{\prime}$, and $z^{\prime}$ to the point with coordinates $x, y$, and $z ; n_{\text {Air }}=1$ is the refractive index of air; and $p_{\text {Air }}, q_{\text {Air }}$, and $\ell_{\text {Air }}$ are the optical direction cosines of the ray path portion in air.

To calculate $p_{\text {Air }}, q_{\text {Air }}$, and $\ell_{\text {Air }}$, we will use the law of refraction in the vector form for the border water-air:

$$
\begin{gathered}
p_{\text {Air }}=p_{W}+v_{x} v, \quad q_{\text {Air }}=q_{W}+v_{y} v \\
\ell_{\text {Air }}=\ell_{W}+v_{z} v
\end{gathered}
$$

where $v_{x}=-x^{\prime} / r_{W}, v_{y}=-y^{\prime} / r_{W}$, and $v_{z}=0$ are the coordinates of the unit vector of the normal to cylindrical surface (2) in the point of ray incidence

$$
\begin{aligned}
v= & \sqrt{n_{\text {Air }}^{2}-n_{W}^{2}+\left(p_{W} \nu_{x}+q_{W} \nu_{y}+\ell_{W} \nu_{z}\right)^{2}} \\
& -\left(p_{W} \nu_{x}+q_{W} \nu_{y}+\ell_{W} \nu_{z}\right) .
\end{aligned}
$$

The substitution of expressions (A.2) into formula (A.7) results in relationship

$$
\begin{aligned}
v= & \frac{1}{r_{W}} \sqrt{r_{W}^{2}\left(1-n_{W}^{2} \cos ^{2} \Theta\right)-y_{A}^{2} n_{W}^{2} \sin ^{2} \varphi \sin ^{2} \Theta} \\
& -\frac{n_{W}}{r_{W}} \sin \Theta \sqrt{r_{W}^{2}-y_{A}^{2} \sin ^{2} \varphi .}
\end{aligned}
$$

Let us assume that, at $s_{\text {Air }}=s_{\text {Air, } 1}^{\triangleright}$, formulas (A.5) determine the coordinates of the first (along the ray path) point of intersection of the ray with cylindrical surface (3), whereas, at $s_{\text {Air }}=s_{\text {Air,2 }}^{\triangleright}$, they determine the coordinates of the second (along the ray path) point of intersection of the ray with cylindrical surface (3). After the substitution of expressions (A.5) into formula (3), we arrive at

$$
\begin{gathered}
s_{\text {Air }, 1}^{\triangleright}=\frac{-y^{\prime} q_{\text {Air }}-x^{\prime} p_{\text {Air }}}{\left(p_{\text {Air }}^{2}+q_{\text {Air }}^{2}\right)}-\frac{1}{2} \Delta s_{\text {Air }}^{\triangleright}, \\
s_{\text {Air }, 2}^{\triangleright}=\frac{-y^{\prime} q_{\text {Air }}-x^{\prime} p_{\text {Air }}}{p_{\text {Air }}^{2}+q_{\text {Air }}^{2}}+\frac{1}{2} \Delta s_{\text {Air }}^{\triangleright}=s_{\text {Air }, 1}^{\triangleright}+\Delta s_{\text {Air }}^{\triangleright},
\end{gathered}
$$

where

$$
\Delta s_{\text {Air }}^{\triangleright}=2 \frac{\sqrt{r_{\text {Air }}^{2}\left(p_{\text {Air }}^{2}+q_{\text {Air }}^{2}\right)-\left(x^{\prime} q_{\text {Air }}-y^{\prime} p_{\text {Air }}\right)^{2}}}{p_{\text {Air }}^{2}+q_{\text {Air }}^{2}}
$$

is the length of the ray path in the radiating cylinder.

Using formulas (A.6) and (A.8), the expression (A.11) may be transformed into the following view:

$$
\Delta s_{\text {Air }}^{\triangleright}=\frac{\sqrt{r_{\text {Air }}^{2}\left(1-n_{W}^{2} \cos ^{2} \Theta\right)-y_{A}^{2} n_{W}^{2} \sin ^{2} \varphi \sin ^{2} \Theta}}{1-n_{W}^{2} \cos ^{2} \Theta} .
$$

\section{B. Conditions for Intersection of the Radiating Cylinder with a Ray}

Let us analyze the conditions under which ray $\mathfrak{R}_{A}(\Theta, \varphi)$ intersects the radiating cylinder. These conditions arise from the formulas used for the calculations of ray paths (see Appendix A). As follows from formula (A.4),

$$
r_{W}^{2}-y_{A}^{2} \sin ^{2} \varphi \geq 0
$$

If condition (B.1) is not fulfilled, then ray $\mathfrak{R}_{A}(\Theta, \varphi)$ does not intersect cylindrical surface (2). In accordance with formula (A.8),

$$
r_{W}^{2}\left(1-n_{W}^{2} \cos ^{2} \Theta\right)-y_{A}^{2} n_{W}^{2} \sin ^{2} \varphi \sin ^{2} \Theta \geq 0 .
$$

If condition (B.2) is not fulfilled, then the total internal reflection of ray $\mathfrak{R}_{A}(\Theta, \varphi)$ from the border water/air takes place.

From formula (A.11), we get

$$
r_{\text {Air }}^{2}\left(1-n_{W}^{2} \cos ^{2} \Theta\right)-y_{A}^{2} n_{W}^{2} \sin ^{2} \varphi \sin ^{2} \Theta \geq 0 .
$$

If condition (B.3) is not fulfilled, then ray $\mathfrak{R}_{A}(\Theta, \varphi)$ does not intersect the radiating cylinder. 
As far as $n_{W} \geq 1$, conditions (B.2) and (B.3) may be fulfilled only in the case when $n_{W}^{2} \cos ^{2} \Theta \leq 1$. In the limiting case $\cos \Theta= \pm 1 / n_{W}$ and conditions (B.2) and (B.3) are fulfilled only at $\varphi=0$. Due to design restrictions, $r_{W}>r_{\text {Air }}$; hence, upon the fulfillment of condition (B.3), condition (B.2) is simultaneously fulfilled. At $n_{W}^{2} \cos ^{2} \Theta \leq 1$ and $n_{W} \geq 1$, the condition

$$
0 \leq \frac{1-n_{W}^{2} \cos ^{2} \Theta}{n_{W}^{2} \sin ^{2} \Theta} \leq 1
$$

is fulfilled. Consequently, upon the fulfillment of condition (B.2), the condition (B.1) is simultaneously fulfilled.

Thus, the conditions under which ray $\mathfrak{R}_{A}(\Theta, \varphi)$ intersects the radiating cylinder have the following view:

$$
\frac{\pi}{2}-\Theta_{\lim } \leq \Theta \leq \frac{\pi}{2}+\Theta_{\lim }, \quad-\varphi_{\lim } \leq \varphi \leq \varphi_{\lim }
$$

where

$$
\begin{gathered}
\varphi_{\lim }=\arcsin \sqrt{\frac{r_{\text {Air }}^{2}\left(1-n_{W}^{2} \cos ^{2} \Theta\right)}{y_{A}^{2} n_{W}^{2} \sin ^{2} \Theta}}, \\
\Theta_{\lim }=\arcsin \frac{1}{n_{W}} .
\end{gathered}
$$

\section{Numerical Integration by the Chebyshev Method}

For the determination of $E_{\text {line }}$ and $E_{r}$ values, it is necessary to calculate the integrals

$$
\begin{aligned}
\mathfrak{D}_{1}= & \int_{-1}^{+1} \exp \left[-\alpha \frac{\left(-y_{A}-r_{W}\right) n_{W}}{\sqrt{n_{W}^{2}-u^{2}}}\right] \frac{1}{\sqrt{n_{W}^{2}-u^{2}}} \mathrm{~d} u \\
\mathfrak{D}_{2}= & \int_{-1}^{+1}\left[1-\alpha n_{W} \frac{r_{W} y_{A}+y_{A}^{2}}{r_{W} \sqrt{n_{W}^{2}-u^{2}}}\right] \\
& \times \exp \left[-\alpha \frac{\left(-y_{A}-r_{W}\right) n_{W}}{\sqrt{n_{W}^{2}-u^{2}}}\right] \frac{1-u^{2}}{\sqrt{\left(n_{W}^{2}-u^{2}\right)^{3}}} \mathrm{~d} u .
\end{aligned}
$$

When the Chebyshev method is used, the formulas for computation of integrals (C.1) have the following view:

$$
\begin{array}{r}
\mathfrak{D}_{1}=\mathfrak{G}_{1}\left\{2 \exp \left[-\alpha \frac{\left(-y_{A}-r_{W}\right) n_{W}}{\sqrt{n_{W}^{2}-\mathfrak{u t}^{2}}}\right]\right. \\
\left.+\exp \left[-\alpha\left(-y_{A}-r_{W}\right)\right]\right\},
\end{array}
$$

where

$$
\begin{aligned}
& \mathfrak{G}_{1}=\frac{1}{3} \int_{-1}^{+1} \frac{1}{\sqrt{n_{W}^{2}-u^{2}}} \mathrm{~d} u=\frac{2}{3} \arctan \left(\frac{1}{\sqrt{n_{W}^{2}-1}}\right), \\
& \mathfrak{u}=\sqrt{\frac{1}{2 \mathfrak{G}_{1}} \int_{-1}^{+1} \frac{u^{2}}{\sqrt{n_{W}^{2}-u^{2}}} \mathrm{~d} u} \\
& =\frac{\sqrt{3}}{2} \cdot \sqrt{\frac{n_{W}^{2} \arcsin \left(1 / n_{W}\right)-\sqrt{n_{W}^{2}-1}}{\arcsin \left(1 / n_{W}\right)}}, \\
& \mathfrak{D}_{2}=\mathfrak{G}_{2}\left\{2\left[1-\alpha n_{W} \frac{r_{W} y_{A}+y_{A}^{2}}{r_{W} \sqrt{n_{W}^{2}-\mathfrak{b}^{2}}}\right]\right. \\
& \times \exp \left[-\alpha \frac{\left(-y_{A}-r_{W}\right) n_{W}}{\sqrt{n_{W}^{2}-\mathfrak{v}^{2}}}\right] \\
& +\left[1-\alpha \frac{r_{W} y_{A}+y_{A}^{2}}{r_{W}}\right] \\
& \left.\times \exp \left[-\alpha\left(-y_{A}-r_{W}\right)\right]\right\} \text {, } \\
& \mathfrak{G}_{2}=\frac{1}{3} \int_{-1}^{+1} \frac{1-u^{2}}{\sqrt{\left(n_{W}^{2}-u^{2}\right)^{3}}} \mathrm{~d} u \\
& =\frac{2}{3} \cdot \frac{-n_{W}^{2}+\arcsin \left(1 / n_{W}\right) n_{W}^{2} \sqrt{n_{W}^{2}-1}+1}{n_{W}^{2} \sqrt{n_{W}^{2}-1}}, \\
& \mathfrak{v}=\sqrt{\frac{1}{2 \mathfrak{G}_{2}} \int_{-1}^{+1} \frac{u^{2}-u^{4}}{\sqrt{\left(n_{W}^{2}-u^{2}\right)^{3}}} \mathrm{~d} u} \\
& =\frac{\sqrt{3}}{2} \cdot\left(n _ { W } ^ { 2 } \left(3-3 n_{W}^{2}+3 \arcsin \left(\frac{1}{n_{W}}\right) n_{W}^{2}\right.\right. \\
& \left.\times \sqrt{n_{W}^{2}-1}-2 \arcsin \left(\frac{1}{n_{W}}\right) \sqrt{n_{W}^{2}-1}\right) \\
& \left.\times\left(-n_{W}^{2}+\arcsin \left(\frac{1}{n_{W}}\right) n_{W}^{2} \sqrt{n_{W}^{2}-1}+1\right)^{-1}\right)^{1 / 2} .
\end{aligned}
$$

\section{Conflict of Interests}

With the submission of this paper, the authors would like to undertake that the representative of the Joint Stock Company "Svarog" (JSC "Svarog") is fully aware of this submission. The management of the JSC "Svarog" considers that the publication of specially selected data of the performed scientific investigations is of immediate interest for the readers of the paper and that it will not cause any prejudice to the JSC "Svarog." 


\section{References}

[1] W. Masschelein and R. G. Rice, Ultraviolet Light in Water and Wastewater Sanitation, Lewis Publishers, 2002.

[2] C. Reichl, C. Buchner, G. Hirschmann, R. Sommer, and A. Cabaj, "Development of a simulation method to predict UV disinfection reactor performance and comparison to biodosimetric measurements," in Proceedings of the Conference on Modelling Fluid Flow, Budapest University of Technology and Economics, pp. 591-598, Budapest, Hungary, 2006.

[3] J. E. Duran, F. Taghipour, and M. Mohseni, "Irradiance modeling in annular photoreactors using the finite-volume method," Journal of Photochemistry and Photobiology A: Chemistry, vol. 215, no. 1, pp. 81-89, 2010.

[4] C. Buchner, Modelling of UV disinfection reactors by means of computational fluid dynamics [M.S. thesis], TU-Wien Atominstitut der Österreichischen Universitäten, Wien, Austria, 2006.

[5] D. Liu, Numerical simulation of UV disinfection reactors: impact of fluence rate distribution and turbulence modeling [Ph.D. thesis], Department of Civil, Construction, and Environmental Engineering, Faculty of North Carolina State University, 2004.

[6] J. R. Bolton, "Calculation of ultraviolet fluence rate distributions in an annular reactor: significance of refraction and reflection," Water Research, vol. 34, no. 13, pp. 3315-3324, 2000.

[7] O. M. Alfano, R. L. Romero, and A. E. Cassano, "Radiation field modelling in photoreactors-I. Homogeneous media," Chemical Engineering Science, vol. 41, no. 3, pp. 421-444, 1986.

[8] P. R. Harris and J. S. Dranoff, "A study of perfectly mixed photochemical reactors," American Institute of Chemical Engineers Journal, vol. 11, no. 3, pp. 497-502, 1965.

[9] S. Jin, K. G. Linden, J. Ducoste, and D. Liu, "Impact of lamp shadowing and reflection on the fluence rate distribution in a multiple low-pressure UV lamp array," Water Research, vol. 39, no. 12, pp. 2711-2721, 2005.

[10] G. E. Imoberdorf, F. Taghipour, and M. Mohseni, "Radiation field modeling of multi-lamp, homogeneous photoreactors," Journal of Photochemistry and Photobiology A: Chemistry, vol. 198, no. 2-3, pp. 169-178, 2008.

[11] E. R. de Bernardez, M. A. Claria, and A. E. Cassano, "Chemical industries. Chemical reaction and reactor engineering," in Analysis and Design of Photoreactors, vol. 26, chapter 13, pp. 839921, CRC Press, 1987.

[12] S. M. Jacob and J. S. Dranoff, "Radial scale-up of perfectly mixed photochemical reactors," Chemical engineering progress symposium series, vol. 62, pp. 47-55, 1966.

[13] S. M. Jacob and J. S. Dranoff, "Light intensity profiles in a perfectly mixed photoreactor," AIChE Journal, vol. 16, no. 3, pp. 359363,1970 .

[14] G. Kirchhoff, "Ueber das Verhältniss zwischen dem Emissionsverm ogen und dem Absorptionsvermögen der Körper fur Wärme und licht," Annalen der Physik und Chemie, vol. 109, no. 4, pp. 275-301, 1860.

[15] R. Clausius, "Ueber die concentration von wärme- und Lichtstrahlen und die gränzen ihrer wirkung," Annalen der Physik, vol. 197, no. 1, pp. 1-44, 1864.

[16] R. Straubel, "Ueber einen allgemeinen Satz der geometrischen Optik und einige Anwendungen," Verhandlungen der Deutschen Physikalischen Gesellschaft, vol. 4, no. 18, pp. 328-334, 1902.
[17] M. Labussière, "Sur l'existence geometrique d'un invariant general des faisceaux de rayons se refractant suivant la loi de descarte et ses applications a l'optique géométrique et au rayonnement," Comptes Rendus Hebdomadaires des Séances, vol. 174, pp. 675-677, 1922.

[18] P. Bouguer, Traité d’optique sur la Gradation de la Lumière, De l'Imprimerie de H. L. Guerin \& L. F. Delatour, Paris, France, 1760.

[19] P. L. Tchebychef, "Sur les quadratures", in Oeuvres de P.L. Tchebychef, A. A. Markov and N. Sonin, Eds., vol. 2, pp. 165180, Commissionnaires de l'Académie Impériale des Sciences, St. Pétersbourg, Russia, 1907.

[20] M. Daimon and A. Masumura, "Measurement of the refractive index of distilled water from the near-infrared region to the ultraviolet region," Applied Optics, vol. 46, no. 18, pp. 3811-3820, 2007.

[21] Australian Water Quality Centre, "Determination of the efficiency of inactivation of MS2 phage, Poliovirus, Cryptosporidium parvum and a bacterial cocktail in adelaide drinking water-a detailed evaluation," Tech. Rep., Australian Water Quality Centre, 2008. 

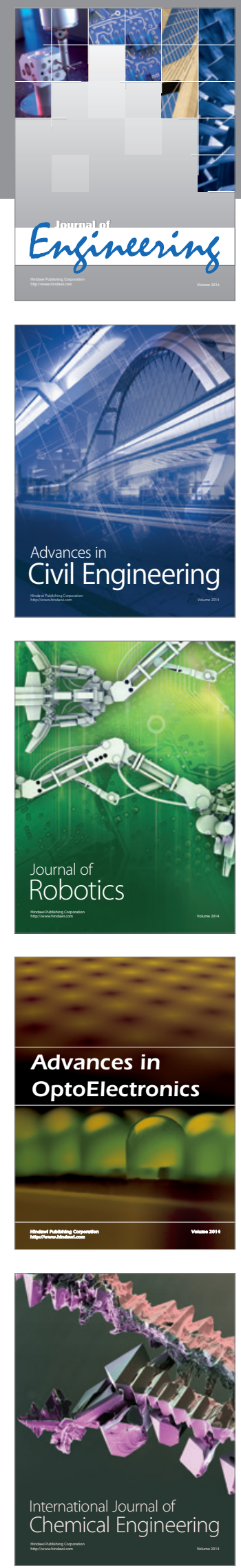

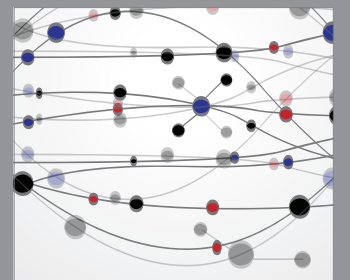

The Scientific World Journal
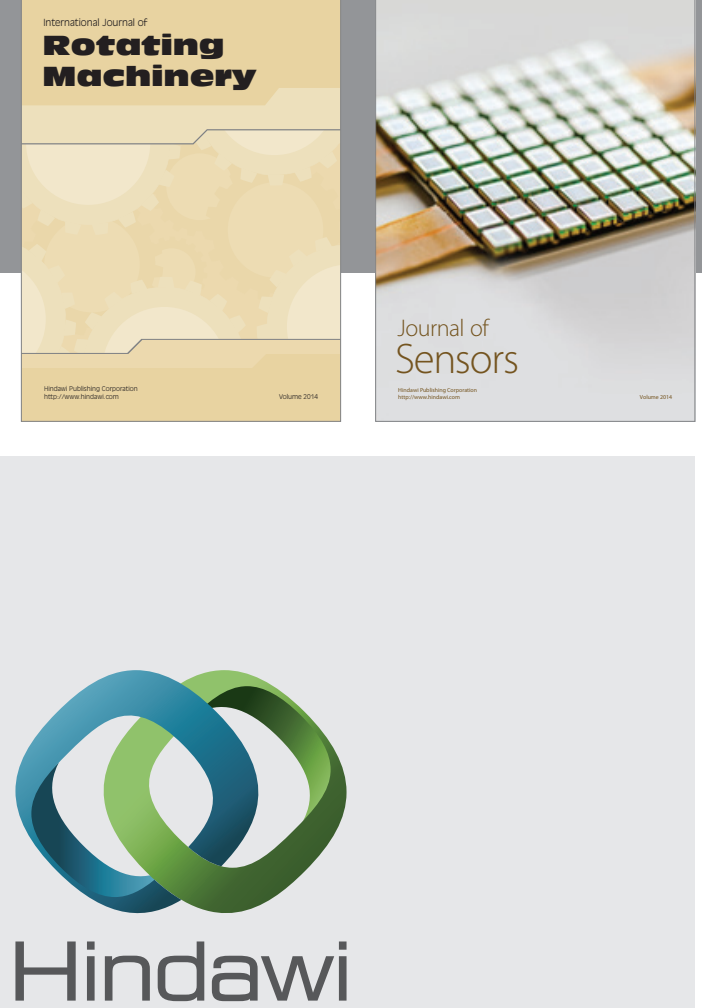

Submit your manuscripts at http://www.hindawi.com
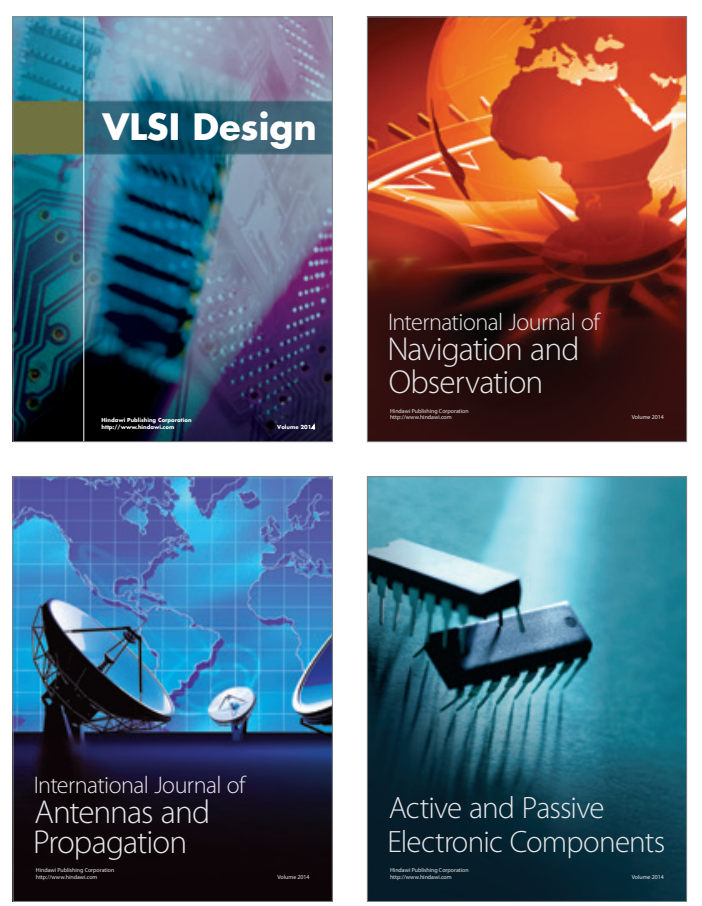
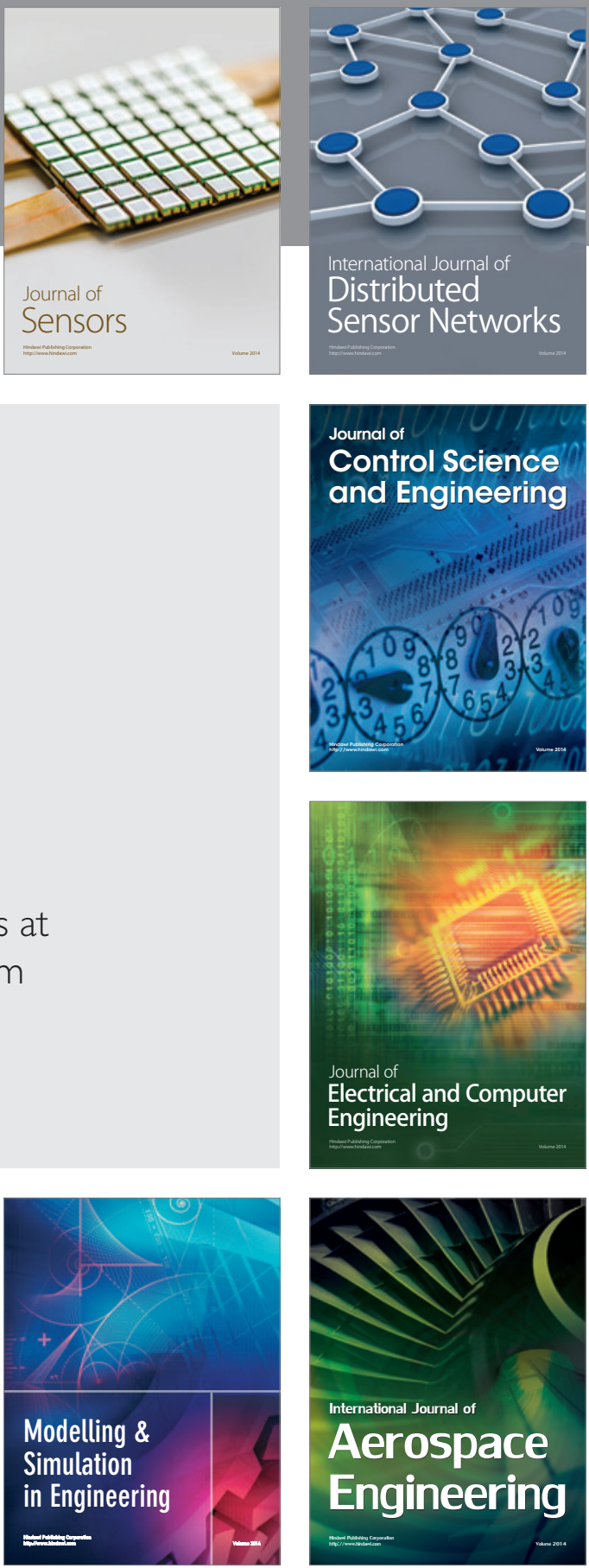

Journal of

Control Science

and Engineering
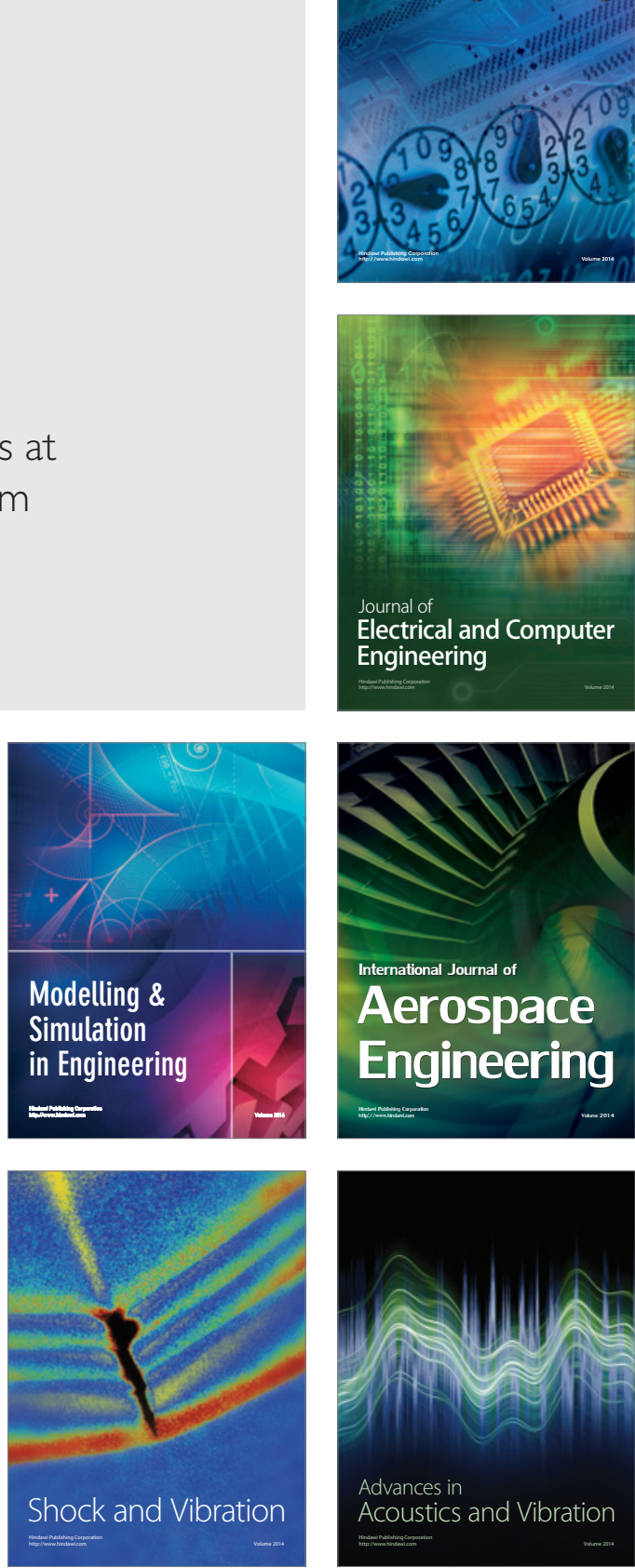